\title{
Normal-phase high-performance liquid chromatographic separation of procyanidins from cacao beans and grape seeds
}

\author{
J. Rigaud*, M.T. Escribano-Bailon ${ }^{\not}$, C. Prieur, J.-M. Souquet and V. Cheynier \\ Institut National de la Recherche Agronomique, Institut des Produits de la Vigne, Laboratoire des Polymères et des Techniques \\ Physico-Chimiques, 2 Place Viala, 34060 Montpellier Cedex (France)
}

(First received June 10th, 1993; revised manuscript received August 11th, 1993)

\begin{abstract}
ABSTRACI
An HPLC method ușing a normal-phase silica column and a gradient of dichloromethane-methanol-formic acid-water mixtures as the eluent was developed to separate procyanidins on a molecular mass basis, without derivatization. It was successfully applied to the analysis of procyanidin extracts from cacao beans and grape seeds. The monomers and major dimers were resolved as discrete peaks. Oligomeric and polymeric components were eluted in order of increasing degree of polymerization, as confirmed by determining the average molecular mass of successive fractions collected from the normal-phase column using gel permeation chromatography, after acetylation.
\end{abstract}

\section{INTRODUCTION}

Proanthocyanidins (i.e., condensed tannins) form a class of compounds that are both ubiquitous and very important owing to their chemical properties. Their tendency to link with proteins is responsible for the astringency of many foods and drinks [1], haze formation in beer [2] and the poor digestibility of fodder legumes [3]. In addition, the oligomers are bitter [1] and show toxicity to methane bacteria [4]. Their pharmaceutical effects are interesting because they act beneficially on the circulatory system [5] and are efficient free radical scavengers [6,7]. All these properties largely depend on their structures and particularly on their degree of polymerization. As a consequence, many methods

\footnotetext{
* Corresponding author.

"Present address: Departamento de Quimica Analitica, Nutricion y Bromatologia, Universidad de Salamanca, Avda. del Campo Charro s/n, 37007 Salamanca, Spain.
}

have been developed to evaluate oligomeric and polymeric ratios.

Among these methods, counter-current chromatography allows the isolation of groups of constituents differing in their chain length [8]. Chromatography on Sephadex LH-20 [9] or Fractogel [10] columns permits the separation of oligomeric fractions, but beyond the tetrameric forms elution requires acetone so that UV monitoring becomes impossible. Gel permeation of the acetylated derivatives makes it possible to obtain a mass profile [11] but requires fairly tedious derivatization procedures that do not allow good recoveries of the samples. Another procedure is to separate procyanidins according to their affinities for proteins [12], but this may be relatively specific and not strictly related to the molecular mass.

Good separations based on molecular mass were achieved with a silica phase but, as it was used in TLC, the resolution was limited to oligomers up to the heptameric forms [13]. When used in HPLC, silica yielded only two rough 
fractions, one containing oligomers and the other polymers [14]. Another polar HPLC column, containing a cyano-bonded silica phase, permitted the separation of peracetylated derivatives [15] or native procyanidins [16]. In the latter instance, many oligomeric groups were distinguishable. However, no individual compound could be separated. In contrast, individual resolution of monomers and many small oligomers was achieved by reversed-phase HPLC $[17,18]$ but the elution order appeared unrelated to the degree of polymerization. However, polymeric forms were separated from the oligomers and eluted towards the end of the chromatogram as a broad, unresolved peak $[19,20]$.

The purpose of this investigation was to develop a chromatographic method to separate oligomeric and polymeric procyanidins on a molecular mass basis. The method had to be suitable both for analytical purposes and for quantitative isolation of procyanidin fractions. Therefore, a normal-phase HPLC method was adapted from the TLC method described by Lea [13]. In particular, the original elution system was modified so as to render it convenient both for UV detection and for the recovery of the eluted compounds.

\section{EXPERIMENTAL}

\section{Chemicals}

All chromatographic solvents were of HPLC grade, purchased from Merck (Darmstadt, Germany) or Prolabo (Paris, France). Acetic anhydride and pyridine were purchased from Labosi (Paris, France) and gallic acid, (+)-catechin and (-)-epicatechin from Sigma (St. Louis, MO, USA). Epicatechin gallate and procyanidin dimers and trimers were isolated from grape seeds in our laboratory and epicatechin tetramer was prepared from cacao beans by Dr. E. Cros (IRCC, Montpellier, France). Polystyrene standards were purchased from Interchim (Montluçon, France).

\section{Extraction}

The cacao procyanidin extract, kindly provided by Dr. E. Cros, had been obtained from lyophilized beans by the following procedure: washing with pentane (lipid removal), extraction with $70 \%$ methanol, filtration, evaporation and dissolution in $60 \%$ acetone, salting out with $\mathrm{NaCl}$, concentration of the organic layer, addition of water, washing with chloroform to remove caffeine and theobromine and extraction with ethyl acetate. After concentration, the resulting extract is brownish red owing to the presence of anthocyanins.

Grape seeds were obtained from frozen berries of Vitis vinifera var. Tannat, harvested at commercial maturity. They were ground under liquid nitrogen, extracted with $60 \%$ acetone and centrifuged. The acetone supernatant was then evaporated to dryness under vacuum, the residue was dissolved in methanol and the solution was centrifuged to obtain a crude extract of procyanidins.

\section{HPLC conditions}

The HPLC apparatus was a Millipore-Waters (Milford, MA, USA) system composed of a U6K injector, a Model 660 gradient controller, two Model 510 pumps and a Model 990 photodiode array detector. The column was a LiChrospher Si 100 (particle size $5 \mu \mathrm{m} ; 250 \times 4 \mathrm{~mm}$ I.D.) purchased from Merck, protected with a guard column $(20 \times 4 \mathrm{~mm}$ I.D.) packed with the same material. The solvents were dichloromethanemethanol-formic acid-water with volume ratios of (A) 5:43:1:1 and (B) 41:7:1:1. The elution conditions were as follows: flow-rate, $1 \mathrm{ml} / \mathrm{min}$; oven temperature, $30^{\circ} \mathrm{C}$; elution, linear gradients from 0 to $20 \% \mathrm{~A}$ in $30 \mathrm{~min}$, from 20 to $50 \% \mathrm{~A}$ in $30 \mathrm{~min}$ and from 50 to $100 \% \mathrm{~A}$ in $5 \mathrm{~min}$, followed by isocratic elution with $100 \%$ A for 5 min and re-equilibration of the column.

\section{Gel permeation chromatography}

Gel permeation chromatography (GPC) was performed on the fractions acetylated with acetic anhydride-pyridine as described by Williams et al. [11]. The GPC analyses were carried out on a Millipore-Waters system, using TSK G $2500 \mathrm{Hxl}$ and TSK G $3000 \mathrm{Hxl}$ columns (particle size 6 $\mu \mathrm{m}$; $300 \times 7.8 \mathrm{~mm}$ I.D.) purchased from Tosohaas (Philadelphia, PA, USA), connected in series. The acetylated compounds were eluted isocratically with tetrahydrofuran (flow-rate 1 
$\mathrm{ml} / \mathrm{min}$; column temperature $30^{\circ} \mathrm{C}$ ) and detected with a Waters M440 detector set at $254 \mathrm{~nm}$. The GPC system was calibrated with eleven polystyrene standards (molecular mass 162-50 000).

\section{Enzymatic hydrolysis}

Enzymatic hydrolysis was performed by incubating the crude grape seed procyanidin extract in $0.1 M$ acetate buffer ( $\mathrm{pH}$ 5.3) with tannase AJ (Gist Brocades, Seclin, France) for $30 \mathrm{~min}$ at $25^{\circ} \mathrm{C}$. The ratio of procyanidins to tannase was close to 10 on a mass basis. Protein and salt were eliminated by percolating the mixture through a Sephadex LH-20 column and washing with water. Degalloylated procyanidins were then eluted with acetone-water (60:40), evaporated to dryness and the residue was dissolved in methanol. It was checked that application of the same purification process to the original crude extract extract did not modify its chromatographic profile.

\section{Procyanidin identification}

The main individual peaks were collected in several runs, pooled, gently evaporated to dryness, with addition of water to prevent hydrolysis due to formic acid concentration, and identificd by micro-thiolysis as described previously [21] and/or enzymatic hydrolysis. The results were confirmed by reversed-phase HPLC [20] and coinjection of the procyanidin extract with pure compounds previously isolated and identified in our laboratory [20] on the normal-phase column.

\section{RESULTS AND DISCUSSION}

Fig. 1 shows the chromatogram of the cacao bean extract. The major components were identified as (-)-epicatechin and its $\mathrm{C}_{4}-\mathrm{C}_{8}$ linked oligomers up to the pentamer. This example illustrates the expected ability of the normalphase HPLC system used to separate oligomeric procyanidins (at least in the epicatechin series) according to their degree of polymerization.

Fig. 2 presents the chromatograms obtained by injection of the grape seed extract on to the normal-phase HPLC system, (A) before and (B) after enzymatic hydrolysis. Identification of the major oligomers (listed in Table I) confirms that,

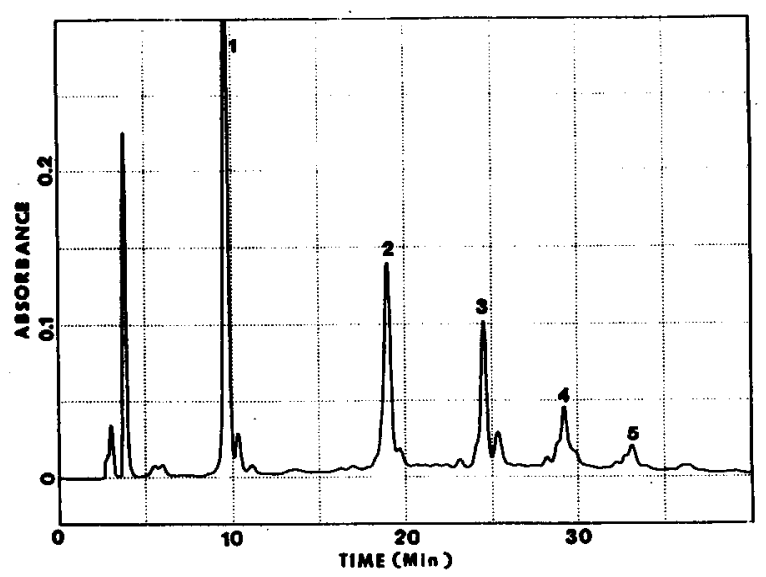

Fig. 1. HPLC analysis of a cacao bean procyanidin extract, monitored at $280 \mathrm{~nm}$. Peaks: $1=$ epicatechin; 2, 3, 4 and $5=$ dimer, trimer, tetramer and pentamer, respectively, of the epicatechin series, tentatively identified by thiolysis.
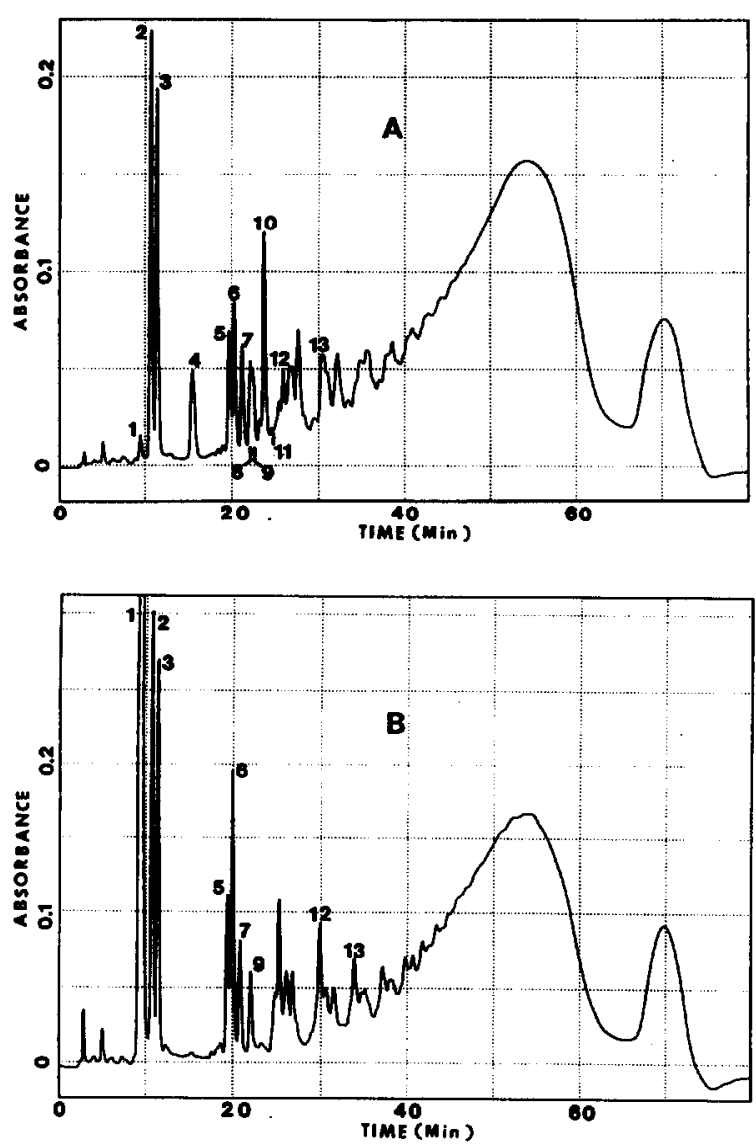

Fig. 2. HPLC analyses (monitored at $280 \mathrm{~nm}$ ) of a grape seed procyanidin extract (A) before and (B) after hydrolysis with tannase. For identification of peaks, see Table $I$. 
TABLE I

LIST OF IDENTIFIED COMPOUNDS

\begin{tabular}{|c|c|}
\hline No. & Compound \\
\hline 1 & Gallic acid \\
\hline 2 & Epicatechin \\
\hline 3 & Catechin \\
\hline 4 & Epicatechin 3-O-gallate \\
\hline 5 & Procyanidin B4 [catechin- $(4 \alpha \rightarrow 8)$-epicatechin] \\
\hline 6 & Procyanidin B2 [epicatechin- $(4 \beta \rightarrow 8)$-epicatechin] \\
\hline 7 & Procyanidin B3 [catechin- $(4 \alpha \rightarrow 8)$-catechin] \\
\hline 8 & Procyanidin B4 3'-O-gallate [catechin- $(4 \alpha \rightarrow 8)$-epicatechin-3-O-gallate] \\
\hline 9 & Procyanidin B1 [epicatechin- $(4 \beta \rightarrow 8)$-catechin] \\
\hline 10 & Procyanidin B2 3-O-gallate [epicatechin-3-O-gallate- $(4 \beta \rightarrow 8)$-epicatechin] \\
\hline 11 & Procyanidin B2 3,3'-di-O-gallate [epicatechin-3-O-gallate- $(4 \beta \rightarrow 8)$-epicatechin-3-O-gallate] \\
\hline 12 & Procyanidin $C 1$ [epicatechin- $(4 \beta \rightarrow 8)$-epicatechin- $(4 \beta \rightarrow 8)$-epicatechin] \\
\hline 13 & Tetramer [epicatechin- $(4 \beta \rightarrow 8)$-epicatechin- $(4 \beta \rightarrow 8)$-epicatechin- $(4 \beta \rightarrow 8)$-epicatechin] \\
\hline
\end{tabular}

although the grape seed extract contained catechin oligomers and mixed catechin-epicatechin derivatives in addition to the epicatechin oligomers found in the cacao extract, they were eluted in successive groups corresponding to increasing degree of polymerization up to the tetramer series. Note that, when co-injected with the grape seed procyanidin extract, the $C_{4}-C_{6}$ linked procyanidin dimers $\mathrm{B} 5$ and $\mathrm{B} 7$ co-eluted with the corresponding $\mathrm{C}_{4}-\mathrm{C}_{8}$ linked procyanidins B2 and B1.

Hydrolysis with tannase (Fig. 2B) improved the resolution of these consecutive groups, suggesting that some galloylated compounds overlapped with the neighbouring fractions in the original sample. In fact, all identified galloylated compounds eluted further than the corresponding non-galloylated compounds. In particular, epicatechin 3-O-gallate (peak 4) eluted between monomers and dimers and B2 $3^{\prime}$-O-gallate (peak 10) between dimers and trimers. Moreover, B2 3,3'-di-O-gallate (peak 11) almost coeluted with the trimers, confirming that some overlapping took place as the complexity of the molecules, and especially the number of galloyl substituents, increased.

Fractions eluting further than the tetramers gradually fused into two broad, unresolved humps. This is probably due to the larger diversi- ty of structures as the degree of polymerization increases, but it may also mean that larger galloylated procyanidin oligomers and polymers are more resistant to hydrolysis by tannase because of enzyme inhibition by tannins or steric hindrance.

In order to check the ability of the silica column to separate larger procyanidins according to their degree of polymerization, four fractions of the eluate were collected. All of them exhibited an absorption maximum near $280 \mathrm{~nm}$ and gave a positive response when heated with butanol- $\mathrm{HCl}$ as described by Bate-Smith [22], confirming that they were mostly composed of tannins. After elimination of the dichloromethane and methanol by rotary evaporation under vacuum, each fraction was submitted to Sephadex LH-20 chromatography to remove formic acid and avoid acid hydrolysis during concentration. The procyanidin fractions were recovered from the Sephadex LH-20 column by elution with acetone-water (60:40). After evaporation to dryness and acetylation, they were analysed by GPC and their average molecular masses were determined by comparison with polystyrene standards. The results obtained for each fraction are presented in Table II along with the calculated average degree of polymerization and elution time in normal-phase HPLC. 
TABLE II

CHARACTERIZATION OF PROCYANIDIN FRACTIONS

\begin{tabular}{lllll}
\hline Parameter & \multicolumn{4}{l}{ Elution time on normal-phase column (min) } \\
\cline { 2 - 5 } & $30-40$ & $40-50$ & $50-65$ & $65-75$ \\
\hline $\begin{array}{l}\text { Average molecular mass } \\
\text { of acetylated fractions }\end{array}$ & 1900 & 3750 & 7200 & 9150 \\
$\begin{array}{l}\text { Average degree } \\
\text { of condensation }\end{array}$ & 3.8 & 7.5 & 14.4 & 18.3 \\
\hline
\end{tabular}

${ }^{a}$ Determined by GPC.

As expected, the degree of polymerization increased with increasing retention time, starting from $\mathrm{ca} .4$ for the fraction eluting at $30 \mathrm{~min}$. The complexity of the polymeric group is responsible for the broad peak at $55 \mathrm{~min}$. The second broad peak eluting near $70 \mathrm{~min}$ corresponds to the largest compounds detected. Note that, for the first fraction collected, the average molecular mass determined by GPC was slightly lower than expected. This may be due to partial hydrolysis of the compounds during concentration prior to acetylation or to contamination of the fraction by lower molecular mass galloylated compounds.

\section{CONCLUSIONS}

The use of a normal-phase silica HPLC column is a good way to achieve elution of procyanidins according to their degree of polymerization. The technique developed permits the separation and determination of oligomeric and polymeric procyanidins from grape seeds. It also allows monomers and many oligomers to be determined individually. The procedure is simple and, as it does not require derivatization of the samples, is suitable for the quantitative isolation of tannin fractions differing in their degree of polymerization. In addition, the amounts of the various procyanidin groups can be determined from their respective peak areas. Therefore, the method should be of help in understanding the role of the different types of procyanidins and monitoring their changes during wine making during wine making and ageing. Finally, it may presumably be extended to procyanidins from other plant sources, although this might require some adaptation if prodelphinidins or other proanthocyanidins are also present.

\section{REFERENCES}

1 A.G.H. Lea and G.M. Arnold, J. Sci. Food Agric., 29 (1978) 478-483.

2 J.W. Gramshaw, J. Inst. Brew., 75 (1969) 61-83.

3 L.Y. Foo, W.T. Jones, L.J. Porter and V.M. Williams, Phytochemistry, 21 (1982) 933-935.

4 J.A. Fields, S. Kortekaas and G. Lettinga, Biol. Wastes, 29 (1989) 241-262.

5 J. Masquelier, in C.R. Symposium Int. " $L$ 'Alimentation et la Consommation de Vin", Verona, Italy, 1982, pp. 147155.

6 S. Uchida, R. Edamatsu, M. Miramatsu, A. Mori, G. Nonaka, I. Nishioka, M. Miwa and M. Ozaki, Med. Sci. Res., 91 (1987) 831-832.

7 J.M. Ricardo da Silva, N. Darmon, Y. Fernandez and S. Mitjavila, J. Agric. Food Chem., 39 (1991) 1549-1552.

8 L.J. Putman and L.G. Buttler, J. Chromatogr., 318 (1985) 85-93.

9 A.G.H. Lea and C.F. Timberlake, J. Sci. Food Agric., 25 (1974) 1537-1545.

10 G. Derdelinckx and J. Jerumanis, J. Chromatogr., 285 (1984) 231-244.

11 V.M. Williams, L.J. Porter and R.W. Hemingway, Phytochemistry, 22 (1983) 569-572.

12 H.I. Oh and J.E. Hoff, J. Food Sci., 44 (1979) 87-96.

13 A.G.H. Lea, J. Sci. Food Agric., 29 (1978) 471-477.

14 C.W. Glennie, W.Z. Kaluza and P.J. Van Niekerk, J. Agric. Food Chem., 29 (1981) 965-968.

15 M.V. Piretti and P. Doghieri, J. Chromatogr., 514 (1990) 334-342.

16 E.L. Wilson, J. Sci. Food Agric., 32 (1981) 257-264. 
17 P. Mulkay, R. Touillaux and J. Jerumanis, Cerevisiae, 1 (1981) 29-31.

18 K. Kantz and V.L. Singleton, Am. J. Enol. Vitic., 41 (1990), 223-228.

19 L.J. Putman and L.G. Buttler, J. Agric. Food Chem., 37 (1989) 943-946.
20 J.M. Ricardo da Silva, J. Rigaud, V. Cheynier, A. Cheminat and M. Moutounet, Phytochemistry, 30 (1991) 1259-1264.

21 J. Rigaud, J. Perez-Ilzarbe, J.M. Ricardo da Silva, V. Cheynier, J. Chromatogr., 540 (1991) 401-405.

22 E.C. Bate-Smith, J. Exp. Bot., 4 (1953) 1-9. 